\title{
1.2 Methods of increasing carrying capacity of transport constructions made of metal corrugated structures under operating conditions
}

\subsubsection{Criteria for assessing the reliable operation of transport constructions made of corrugated metal structures}

The assessment of the bearing capacity of transport constructions with MCSs is one of the most important factors in the formation and maintenance of reliable operation of transport infrastructure [11-19]. Therefore, the development and improvement of methods for calculating transport constructions is constantly given considerable attention, especially to promising constructions made of corrugated metal structures.

The safe operation of corrugated metal structures in the body of the embankment of the railway or highway is determined by the ratio of the parameters of the bearing capacity of the structure $R(t)$ and the load $Q(t)$ caused by the action of mobile transport units. The ratio of $R_{0}$ to $Q_{0}$, even before the operation of corrugated metal structures determines the coefficient of strength:

$$
n_{0}=R_{0} / Q_{0} .
$$

The point of intersection of the curves of the bearing capacity $R(t)$ and the magnitude of the load $Q(t)$ gives the calculated durability of the construction $t_{p}$, i.e., determines the time to failure. The safe service life of the construction $[t]$ is defined as the ratio of the service life $t_{p}$ of the construction with MCSs to the safety factor $n_{t}$ :

$$
[t]=t_{p} / n_{t} \text {. }
$$

In formula (2) the value of the safety factor $n_{t}$ is determined according to reference materials, expertly or by calculation. In addition, it can be seen from formula (2) that if $n_{0}=$ const (or $Q_{0}=R_{0}=$ const), then the transport construction with MCSs will have unlimited durability: $t_{r}=t_{p}^{(1)}=\infty$. In the case of $n_{0} \neq$ const, the transport construction with MCSs will have limited durability, which depends on the intensity of changes in the load parameters $Q(t)$ and the safety factor $n_{t}$. In addition, durability will depend on the types of cross-sectional shapes of the construction, the size of the 
corrugated metal sheet and the design features of structures such as MultiPlate or SuperCor.

It is obvious that the lowest value of durability of the construction with MCSs corresponds to the case when there is a simultaneous decrease in bearing capacity $R$ and transport load increase $Q$. The maximum durability of the construction with MCSs will be in the case when $Q=$ const and $R=$ const. Therefore, at the design stage of the construction with MCSs it is necessary to ensure their safe operation by reducing the dynamic action of the transport load $Q$ and increasing the bearing capacity $R$, and hence - the durability of the construction.

At present, both in Ukraine and in other countries, there are a number of regulations on calculations of metal transport constructions strength reflecting the current level of knowledge and technical capabilities in this sphere [31]. The calculation of the strength of the ultimate condition of pipelines is performed on the basis of the ultimate strength of the structural material under the following condition:

$$
\sigma_{p} \leq \psi[\sigma]
$$

where: $\sigma_{p}$-the maximum total stress from the action of force factors; $[\sigma]$ - estimated allowable stress values for the construction material; $\psi$ - coefficient that takes into account the stress state of the structure. Permissible stresses are determined by the formula:

$$
[\sigma]=\frac{\sigma_{B} m}{K_{1} K_{2}}
$$

where: $\sigma_{B}-$ the ultimate strength of the material in accordance with state standards and specifications; $m$ - coefficient of working conditions of the construction; $K_{1}-$ safety factor for the material; $K_{2}-$ reliability factor.

The safety factor of the material reflects possible reduction of the ultimate strength of the metal structure compared to the standard value, because during operation of constructions with MCSs the reduction of wall thickness of the structure is possible compared to the design one and, accordingly, reliable operation of the construction with MCSs is questionable. 
The coefficient of working conditions takes into account the possible inconsistency of the selected calculation scheme with the actual operation of the construction, in particular, the features of the interaction of the corrugated metal structure with the environment. In addition, this ratio reflects the impact of the consequences of structure destruction on the cost of repair and restoration works.

The calculation of transport constructions made of corrugated metal structures for strength is performed in accordance with regulations DBN V.1.2-15: 2009 [20] and VBN V.2.3-218-198: 2007 [21] according to the formula:

$$
\frac{N}{A} \leq R_{y} m
$$

where: $N$ - normal (tangential) force in the corrugated structure from design loads per length $\lambda$ of one corrugation; $A$ - cross-sectional area of one wave of corrugation; $R_{y}$ - design resistance of steel beyond yield strength, adopted in accordance with DBN V.2.3-14, and $m=0.9$ - coefficient of operating conditions of the construction with MCSs.

According to [21], the limiting relative changes of the horizontal or vertical diameters of the construction should not exceed $5 \%$. They are determined by the formula:

$$
\varsigma=\frac{\frac{\gamma \cdot(h+R)}{E}}{\frac{\delta}{R}+\frac{E_{0}}{E}\left(1-v^{2}\right)}
$$

where: $R$ - radius of the structure; $E_{0}$ - modulus of strain of the backfill soil; $E-$ modulus of steel elasticity; $\delta$-thickness of the corrugated structure; $v=0,25$-Poisson's ratio of the building material; $\gamma$-specific weight of the building material.

The considered criteria are basic for estimation of the bearing capacity of transport constructions made of metal corrugated structures.

\subsubsection{Reasons for reduced load-bearing capacity and durability of constructions made of metal corrugated structures}


The main disadvantage of constructions made of corrugated metal structures, with increased beams, is the low operational reliability due to various factors of impact $[22,23,24]$. These include the specifics of construction with MCSs operation, which is determined by the nature of interaction of the metal shell and the backfill soil; provision of the necessary shape and certain dimensions with a limited height of the embankment on the railway or highway.

The poor compaction of the soil backfill can cause uneven subsidence of the embankment over the metal corrugated structures, strain of the reinforcement of the embankment slopes and distortion of the cross-sectional shape of constructions with MCSs. The loss of the construction shape has a negative impact on the performance of railways or highways including the construction itself (the appearance of potholes on the carriageway or unevenness on the railway track), and its durability (the appearance of deflections, residual strains).

To ensure the bearing capacity and durability increase of transport constructions with MCSs, it is necessary to conduct quality work both at the design and at the building stages. At the design stage, the deterioration of material properties over time should be taken into account, which is caused by the aggressiveness of the environment (soil, groundwater, chemicals), providing the use of fairly stable materials and their protection. In addition, the axial load from vehicles may increase during operation of the facility. Therefore, the development of design solutions at the design stage, to increase the load-bearing capacity of constructions, with minimal economic investment is an urgent problem and needs immediate solution.

\subsubsection{Constructive options of bearing capacity and durability increase of transport constructions made of metal corrugated structures are offered}

At present, there are a number of technical solutions for the reinforcement of constructions with MCSs. For example, increasing the bearing capacity of MCSs by means of metal sheet thickness enlargement of the structure or by double corrugation. However, the use of such design options is not always effective, both economically and technologically. This is due to the fact that each of the facilities functions in different operating conditions and requires an individual design solution to increase the load- 
bearing capacity. The constructive decision as for the increase of a corrugated sheet thickness is economically unprofitable in most cases, and at each design of a construction, it is necessary to carry out technical and economic calculations.

Therefore, with the purpose of the load-bearing capacity and durability increase, and consequently, the safe operation of constructions with MCSs, the following design options are proposed in this work: the installation of additional stiffeners in the sinuses of a corrugated sheet in the form of round rods with diameter $16 \mathrm{~mm}$. that increase structural rigidity; the installation of reinforcing plates curved in the shape of a corrugated sheet with the thickness equal to the thickness of the metal corrugated structure into the sinuses of the corrugated profile sheet; carrying out double corrugation in places of action of the greatest forces from vehicles.

The proposed design solutions should be taken into account at the design stage of a construction with MCSs. To confirm the effectiveness of the applied solutions, it is necessary to conduct a preliminary evaluation of them, with regard operational loads and economical indices.

A constructive variant of the bearing capacity and durability of MCSs increase by installing metal stiffeners into inside sinuses of the corrugated structure is shown in Fig. 1.

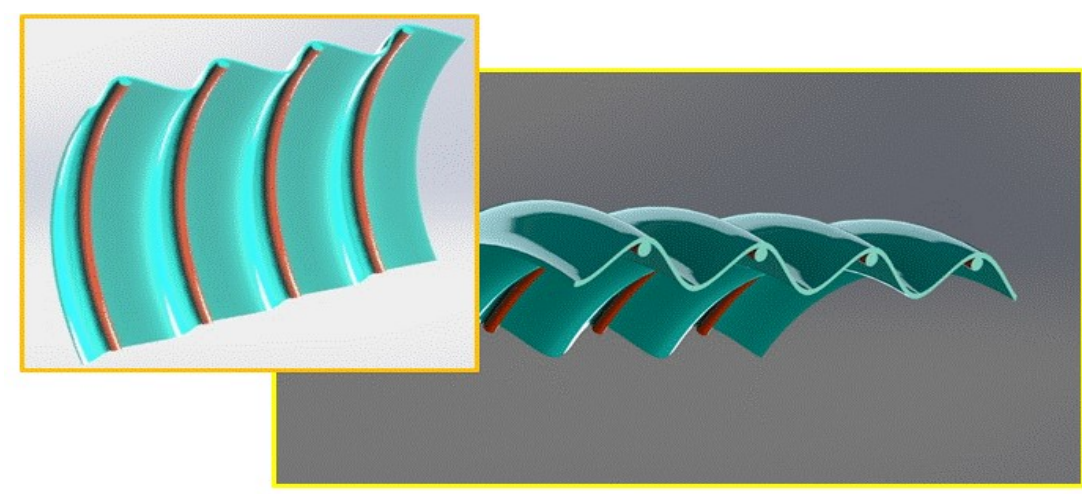

Figure 1. Metal corrugated structure with additional stiffeners

The stiffeners are round rods with diameter $16 \mathrm{~mm}$, which are installed in the convex part of the corrugated structure on the inside. At the same time, it covers all cross section of a construction and works together with a corrugated metal sheet. 
Another way to increase the load-bearing capacity and durability of transport constructions with MCSs is to install an overlay plate along the contour of the corrugated metal sheet (Fig. 2).

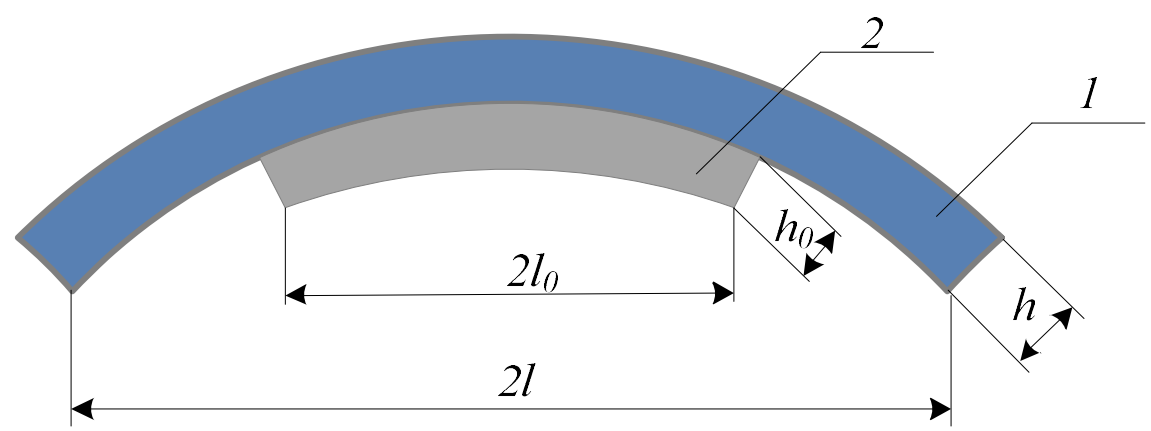

Figure 2. Overlay plate to increase the load-bearing capacity of MCSs: 1 - existing corrugated metal structure; 2 - metal overlay plate

Installation of stiffeners must be carried out in such a way that the reinforcement zone of the construction occupies the width of the carriageway or railway track (Fig. $3)$.

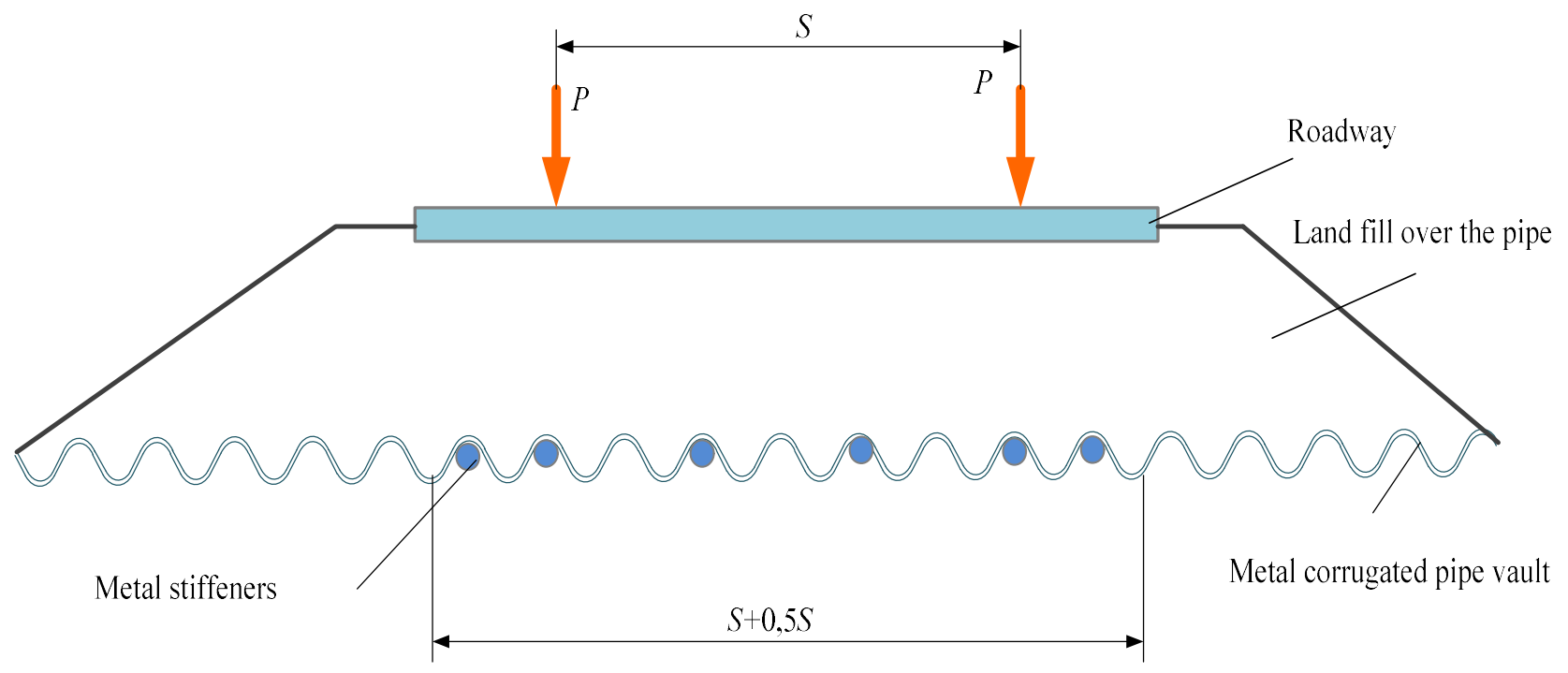

Figure 3. Scheme of reinforcement of the transport construction with MCSs by stiffeners

The advantage of this approach is the ability to distribute the material more efficiently, setting the stiffeners only in the most loaded cross-sectional segments of metal corrugated structures. One of the disadvantages of the design solution is the increase in the complexity of the work during metal structures installation. 


\subsubsection{Reinforcement of the transport construction with MCSs}

by double corrugation

In world practice, the method of double corrugation is used (Fig. 4), which is applied in the building of transport constructions made of corrugated structures such as MultiPlale and SuperCor.

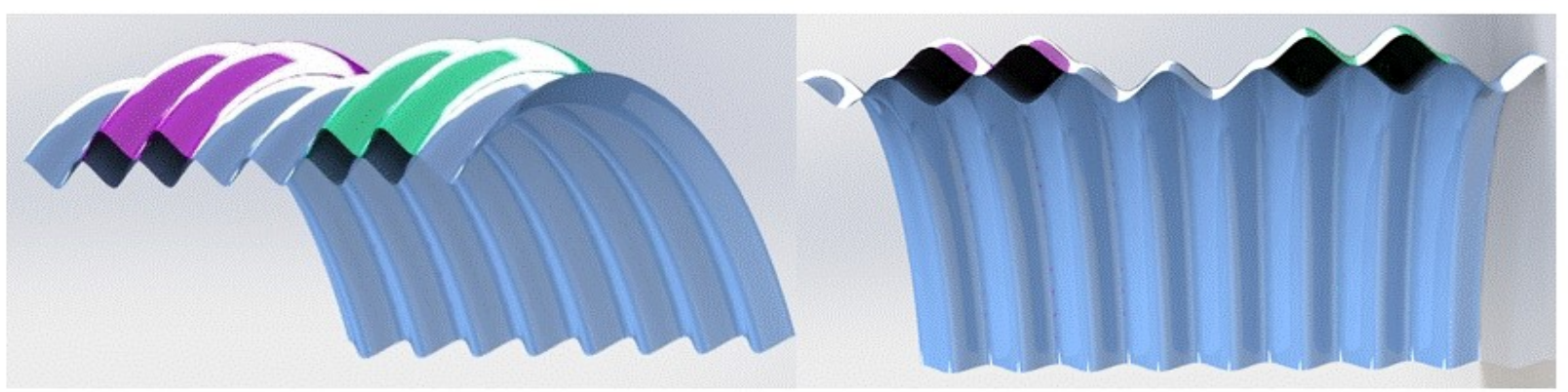

Figure 4. Metal corrugated structure with double corrugation

Double corrugation is not performed along the entire cross section of the pipe or bridge, but only in places where there are the greatest bending moments in the wall of the construction under the action of dynamic transport load. This method allows to increase the load-bearing capacity and durability of corrugated metal structures, and, in addition, to increase the safety traffic of vehicles. However, there are no reliable methods for assessing the load-bearing capacity of double-corrugated structures, which justifies the research in this sphere.

Each of the considered options for increasing the load-bearing capacity and durability of constructions with MCSs has its advantages and disadvantages. To compare and select the optimal design solutions, we will conduct a series of calculations to assess the load-bearing capacity of structures with MCSs reinforced with stiffening ribs as well as technical and economical indices depending on the power action of vehicles.

It should be noted that the proposed design solutions change the calculation scheme of the task and require the development of methods for estimating the stressstrain state. 


\subsubsection{Method for estimating the stress-strain state of a transport construction with MCSs reinforced with round stiffeners}

Let's consider the case of a transport construction reinforcement made of corrugated metal structures by installing round stiffeners in the sinuses of the corrugated pipe profile. The view of the studied design option is shown in Fig. 1.

Mathematical formulation of the research. Let's consider an elastic corrugated cylindrical shell referred to the cylindrical coordinate system $r, \varphi, z(0 \leq \varphi$ $<2 \pi$ ). The cross section of this structure in the direction of $z$ coordinate is shown in Fig.

5 .

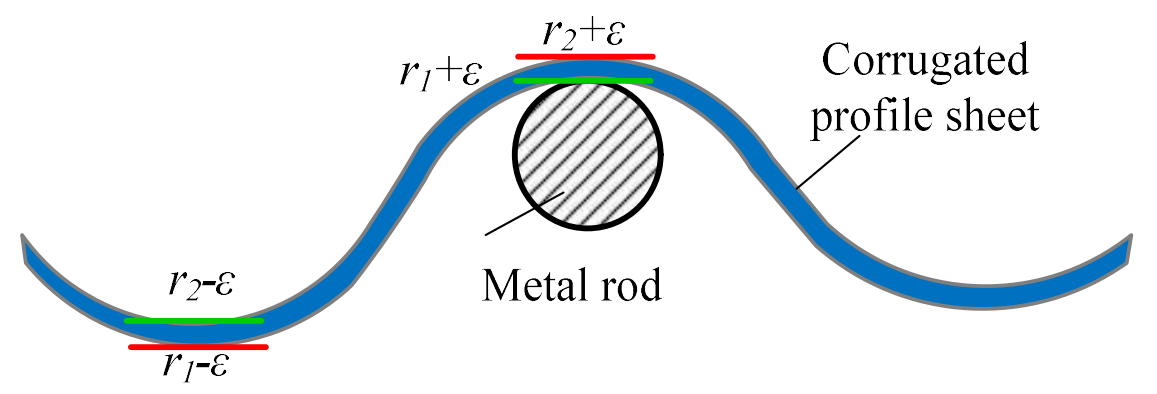

Figure 5. Scheme to describe the mathematical model of a corrugated shell reinforced with ribs

The inner $S_{1}$ and outer $S_{2}$ of the shell surface are respectively described by the equations:

$$
\begin{aligned}
& r=r_{1}+\varepsilon \sin a z ; \\
& r=r_{2}+\varepsilon \sin a z .
\end{aligned}
$$

Shell, along the lines,

$$
\left\{\begin{array}{l}
r=r_{1}+\varepsilon \\
z=\frac{\pi}{a}\left(\frac{1}{2}+2 n\right) \quad(n=0, \pm 1, \pm 2, \ldots)
\end{array}\right.
$$

is reinforced with round stiffeners, which occupy areas:

$$
\left(V_{n}\right)=\left\{(r, \varphi, z): r_{0}-\sqrt{R^{2}-\left(z-z_{0 n}\right)^{2}} \leq r \leq r_{0}+\sqrt{R^{2}-\left(z-z_{0 n}\right)^{2}}, 0 \leq \varphi<2 \pi, z_{0 n}-R \leq z \leq z_{0 n}+R\right\},
$$

where: $R$ - radius of the circle, which is the cross section of the stiffener and $r_{0}$ is equal to: 


$$
r_{0}=r_{1}+\varepsilon-R ; z_{0 n}=\frac{\pi}{a}\left(\frac{1}{2}+2 n\right) .
$$

The considered shell is under the action of forces $P_{r}(\varphi, z), P z(\varphi, z)$ applied to the outer surface $S_{2}$. The inner surface $S_{1}$ and the reinforcing stiffeners, with the exception of lines (9), are load-free. The side surfaces $z= \pm l$ of the shell are also free of loads.

We assume that the conditions of ideal mechanical contact of the shell with stiffeners are satisfied on the lines (9).

To determine the stress state of the shell and stiffeners, we use the equations and relations of the theory of elasticity.

The equations of equilibrium of the elementary volume of the considered bodies have the form:

$$
\begin{aligned}
& \frac{\partial \sigma_{r r}}{\partial r}+\frac{1}{r} \frac{\partial \sigma_{r \varphi}}{\partial \varphi}+\frac{\partial \sigma_{r z}}{\partial z}+\frac{\sigma_{r r}-\sigma_{\varphi \varphi}}{r}=0, \\
& \frac{\partial \sigma_{r \varphi}}{\partial r}+\frac{1}{r} \frac{\partial \sigma_{\varphi \varphi}}{\partial \varphi}+\frac{\partial \sigma_{\varphi z}}{\partial z}+\frac{2 \sigma_{r \varphi}}{r}=0, \\
& \frac{\partial \sigma_{r z}}{\partial r}+\frac{1}{r} \frac{\partial \sigma_{\varphi z}}{\partial \varphi}+\frac{\partial \sigma_{z z}}{\partial z}+\frac{\sigma_{r z}}{r}=0,
\end{aligned}
$$

where $\sigma_{i j}(i, j=r, \varphi, z)$ are the components of the stress tensor.

The components of the strain tensor $\varepsilon_{i j}(i, j=r, \varphi, z)$ and the displacement vector $u_{i}(i=r, \varphi, z)$ are related by the relations:

$$
\begin{gathered}
\varepsilon_{r r}=\frac{\partial u_{r}}{\partial r}, \quad \varepsilon_{\varphi \varphi}=\frac{1}{r}\left(u_{r}+\frac{\partial u_{\varphi}}{\partial \varphi}\right), \quad \varepsilon_{z z}=\frac{\partial u_{z}}{\partial z}, \\
\varepsilon_{r \varphi}=\frac{1}{2}\left(\frac{1}{r} \frac{\partial u_{r}}{\partial \varphi}+\frac{\partial u_{\varphi}}{\partial r}-\frac{u_{\varphi}}{r}\right), \\
\varepsilon_{\varphi z}=\frac{1}{2}\left(\frac{\partial u_{\varphi}}{\partial z}+\frac{1}{r} \frac{\partial u_{z}}{\partial \varphi}\right), \quad \varepsilon_{r z}=\frac{1}{2}\left(\frac{\partial u_{z}}{\partial r}+\frac{\partial u_{r}}{\partial z}\right) .
\end{gathered}
$$

The stresses $\sigma_{i j}$ and the strains $\varepsilon_{i j}$ are related by Hooke's law relations, which have the form: 


$$
\begin{aligned}
& \sigma_{r r}=2 G\left(\varepsilon_{r r}+\frac{v \theta}{1-2 v}\right), \\
& \sigma_{\varphi \varphi}=2 G\left(\varepsilon_{\varphi \varphi}+\frac{v \theta}{1-2 v}\right), \\
& \sigma_{z z}=2 G\left(\varepsilon_{z z}+\frac{v \theta}{1-2 v}\right), \\
& \sigma_{r \varphi}=2 G \varepsilon_{r \varphi}, \sigma_{\varphi z}=2 G \varepsilon_{\varphi z}, \sigma_{r z}=2 G \varepsilon_{r z} .
\end{aligned}
$$

Here: $\theta=\varepsilon_{\mathrm{rr}}+\varepsilon_{\varphi \varphi}+\varepsilon_{\mathrm{zz}} ; G-$ shear modulus; $v$ - Poisson's ratio.

Conditions for the periodicity of the stress-strain state of the shell and stiffeners with respect to the coordinate $\varphi$ are given in the form:

$$
\begin{gathered}
\left.\sigma_{r \varphi}\right|_{\varphi=0}=\left.\sigma_{r \varphi}\right|_{\varphi=2 \pi-0},\left.\quad \sigma_{\varphi \varphi}\right|_{\varphi=0}=\left.\sigma_{\varphi \varphi}\right|_{\varphi=2 \pi-0},\left.\quad \sigma_{\varphi z}\right|_{\varphi=0}=\left.\sigma_{\varphi z}\right|_{\varphi=2 \pi-0}, \\
\left.u_{r}\right|_{\varphi=0}=\left.u_{r}\right|_{\varphi=2 \pi-0},\left.\quad u_{\varphi}\right|_{\varphi=0}=\left.u_{\varphi}\right|_{\varphi=2 \pi-0},\left.\quad u_{z}\right|_{\varphi=0}=\left.u_{z}\right|_{\varphi=2 \pi-0} .
\end{gathered}
$$

The conditions of the ideal mechanical contact of considered bodies (stiffening ribs and a corrugated overlay) have the form:

$$
\begin{aligned}
& \sigma_{r r}\left(r_{1}+\varepsilon-0, \varphi, z_{0 n}\right)=\sigma_{r r}\left(r_{1}+\varepsilon+0, \varphi, z_{0 n}\right), \\
& \sigma_{r \varphi}\left(r_{1}+\varepsilon-0, \varphi, z_{0 n}\right)=\sigma_{r \varphi}\left(r_{1}+\varepsilon+0, \varphi, z_{0 n}\right), \\
& \sigma_{r z}\left(r_{1}+\varepsilon-0, \varphi, z_{0 n}\right)=\sigma_{r z}\left(r_{1}+\varepsilon+0, \varphi, z_{0 n}\right), \\
& u_{r}\left(r_{1}+\varepsilon-0, \varphi, z_{0 n}\right)=u_{r}\left(r_{1}+\varepsilon+0, \varphi, z_{0 n}\right), \\
& u_{\varphi}\left(r_{1}+\varepsilon-0, \varphi, z_{0 n}\right)=u_{\varphi}\left(r_{1}+\varepsilon+0, \varphi, z_{0 n}\right), \\
& u_{z}\left(r_{1}+\varepsilon-0, \varphi, z_{0 n}\right)=u_{z}\left(r_{1}+\varepsilon+0, \varphi, z_{0 n}\right) .
\end{aligned}
$$

Let's assume that the set $S$ corresponds to the union of the surface $S_{1}$ and the surfaces of the stiffeners, from which lines (9) are subtracted. In this case, the conditions at the limits of the shell and the stiffening ribs take the form:

$$
\begin{aligned}
& \left.\left(\sigma_{r r} n_{r}+\sigma_{r z} n_{z}\right)\right|_{S}=0, \\
& \left.\left(\sigma_{r \varphi} n_{r}+\sigma_{\varphi z} n_{z}\right)\right|_{S}=0, \\
& \left.\left(\sigma_{r z} n_{r}+\sigma_{z z} n_{z}\right)\right|_{S}=0,
\end{aligned}
$$




$$
\begin{aligned}
& \left.\left(\sigma_{r r} n_{r}+\sigma_{r z} n_{z}\right)\right|_{S_{2}}=P_{r}(\varphi, z), \\
& \left.\left(\sigma_{r \varphi} n_{r}+\sigma_{\varphi z} n_{z}\right)\right|_{S_{2}}=0, \\
& \left.\left(\sigma_{r z} n_{r}+\sigma_{z z} n_{z}\right)\right|_{S_{2}}=P_{z}(\varphi, z) .
\end{aligned}
$$

The limit conditions at the ends of the metal corrugated pipe will be expressed as follows:

$$
\left.\sigma_{r z}\right|_{z= \pm l}=0,\left.\quad \sigma_{\varphi z}\right|_{z= \pm l}=0,\left.\quad \sigma_{z z}\right|_{z= \pm l}=0,
$$

where $n_{r}, n_{z}$ - directing cosines of the vectors of unit external normals to the surfaces of considered bodies.

Surfaces $S_{1}$ correspond to value:

$$
n_{r}=-\frac{1}{\Delta}, n_{z}=\frac{\varepsilon a \cos a z}{\Delta},
$$

and surfaces $S_{2}$ correspond to value:

$$
n_{r}=\frac{1}{\Delta}, n_{z}=-\frac{\varepsilon a \cos a z}{\Delta} .
$$

In formulas (19) and (20) the value $\Delta$ is determined according to the formula:

$$
\Delta=\sqrt{1+(\varepsilon a \cos a z)^{2}} .
$$

those parts of stiffeners surfaces, which are determined by the equation $r=r_{0}+\sqrt{R^{2}-\left(z-z_{0 n}\right)^{2}}$, correspond to the following values:

$$
\begin{gathered}
n_{r}=\frac{\sqrt{R^{2}-\left(z-z_{0 n}\right)^{2}}}{R}, \\
n_{z}=\frac{z-z_{0 n}}{R},
\end{gathered}
$$

and parts of stiffeners surfaces, which are determined by the equation $r=r_{0}-\sqrt{R^{2}-\left(z-z_{0 n}\right)^{2}}$, correspond to values:

$$
\begin{gathered}
n_{r}=-\frac{\sqrt{R^{2}-\left(z-z_{0 n}\right)^{2}}}{R}, \\
n_{z}=\frac{z-z_{0 n}}{R} .
\end{gathered}
$$


Next, according to the above method, we evaluate the stress-strain state of the construction with different design options to increase its load-bearing capacity and durability.

\subsubsection{Analytical method for estimating the stress-strain state of a}

\section{transport construction with MCSs reinforced with metal stiffening plates}

The next design option to increase the load-bearing capacity and durability of constructions is shown in Fig. 2.

\subsubsection{Mathematical formulation of the research problem}

Let's consider an elastic corrugated cylindrical shell referred to the cylindrical coordinate system $r, \varphi, z \quad(0 \leq \varphi<2 \pi)$. The cross section of this structure in the direction of $z$ coordinate is shown in Fig. 6.

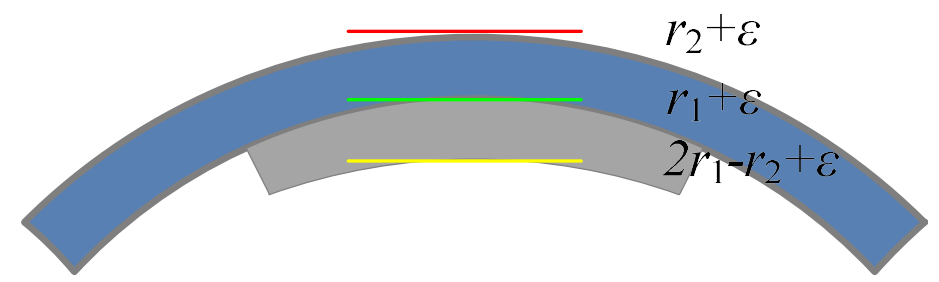

Figure 6. Diagram to describe the mathematical model of the corrugated shell reinforced with plate stiffeners

The inner $S_{1}$ and outer $S_{2}$ of the shell surface are described by equations (7) and (8), respectively.

The shell reinforced with stiffening plates, which occupy the areas:

$$
\left(V_{n}\right)=\left\{(r, \varphi, z): 2 r_{1}-r_{2}+\varepsilon \sin a z \leq r \leq r_{1}+\varepsilon \sin a z ; 0 \leq \varphi<2 \pi, z_{0 n}-l_{0} \leq z \leq z_{0 n}+l_{0}\right\}(n=0, \pm 1, \pm 2 \ldots),
$$

where

$$
z_{0 n}=\frac{\pi}{a}\left(\frac{1}{2}+2 n\right)
$$

The considered shell is under the action of forces $P_{r}(\varphi, z), P_{z}(\varphi, z)$ applied to the outer surface $S_{2}$. The inner surface $S_{1}$ and the reinforcing plates, except for areas

$$
\left(\sum_{n}\right)=\left\{(r, \varphi, z): r=r_{1}+\varepsilon \sin a z ; 0 \leq \varphi<2 \pi ; z_{0 n}-l_{0} \leq z \leq z_{0 n}+l_{0}\right\} \quad(n=0, \pm 1, \pm 2, \ldots)
$$

are free from loads. The side surfaces $z= \pm l$ of the shell and the side surfaces $z=z_{0 n} \pm l_{0}$ of the stiffening plates are also free from loads. 
Let's assume that at the points of areas (27), the conditions of ideal mechanical contact of the shell with the reinforcing plates are fulfilled.

To determine the stress state of the bodies under consideration, we use the above equations and the relations of the theory of elasticity (12)-(14). In this case, the set $S$ is the union of the surface $S_{1}$ and the inner surfaces of the reinforcing plates, from which the areas (27) are subtracted. The conditions on the side surfaces of the reinforcing plates are as follows:

$$
\left.\sigma_{r z}\right|_{z=z_{0 n} \pm l_{0}}=0,\left.\quad \sigma_{\varphi z}\right|_{z=z_{0 n} \pm l_{0}}=0,\left.\quad \sigma_{z z}\right|_{z=z_{0 n} \pm l_{0}}=0 .
$$

According to the conditions of the ideal mechanical contact of considered bodies,

the values

$$
\sigma_{r} n_{r}+\sigma_{r z} n_{z}, \sigma_{r \phi} n_{r}+\sigma_{\varphi q} n_{z}, \sigma_{r z} n_{r}+\sigma_{z} n_{z}, u_{r}, u_{\varphi}, u_{z}
$$

$r=r_{1}+\varepsilon \sin a z-0, z_{0 n}-l_{0} \leq z \leq z_{0 n}+l_{0}$ are equal to the values at $r=r_{1}+\varepsilon \sin a z+0, z_{0 n}-l_{0} \leq z \leq z_{0 n}+l_{0}$. The guide cosines $n_{r}, n_{z}$, which correspond to the surfaces of the reinforcing plates, are calculated in the same way as the guide cosines corresponding to the surfaces of the shell.

\subsubsection{Analytical method for estimating the stress-strain state of a} transport construction with MCSs under condition of double corrugation

\subsubsection{Mathematical formulation of the research}

Another design option is to strengthen the metal corrugated construction with an additional corrugated structure (Fig. 4). To do this, let's consider two elastic corrugated cylindrical shells A and B of equal length, referred to the cylindrical coordinate system $r, \varphi, z(0 \leq \varphi<2 \pi)$. The cross section of this structure in the direction of $z$ coordinate is shown in Fig. 7. The inner $S_{1}$ and outer $S_{2}$ of shell A surfaces, respectively, are described by the equations:

$$
\begin{aligned}
& r=r_{1}+\varepsilon \sin a z ; \\
& r=r_{2}+\varepsilon \sin a z,
\end{aligned}
$$

and inner $S_{3}$ and outer $S_{4}$ surfaces of shell B are described by the equations:

$$
\begin{gathered}
r=2\left(r_{1}-\varepsilon\right)-r_{2}-\varepsilon \sin a z ; \\
r=r_{1}-2 \varepsilon-\varepsilon \sin a z .
\end{gathered}
$$


Along the lines

$$
\left\{\begin{array}{l}
r=r_{1}-\varepsilon \\
z=\frac{\pi}{a}\left(-\frac{1}{2}+2 n\right) \quad(n=0, \pm 1, \pm 2, \ldots)
\end{array}\right.
$$

there is a mechanical contact of shells under consideration, which is regarded ideal.

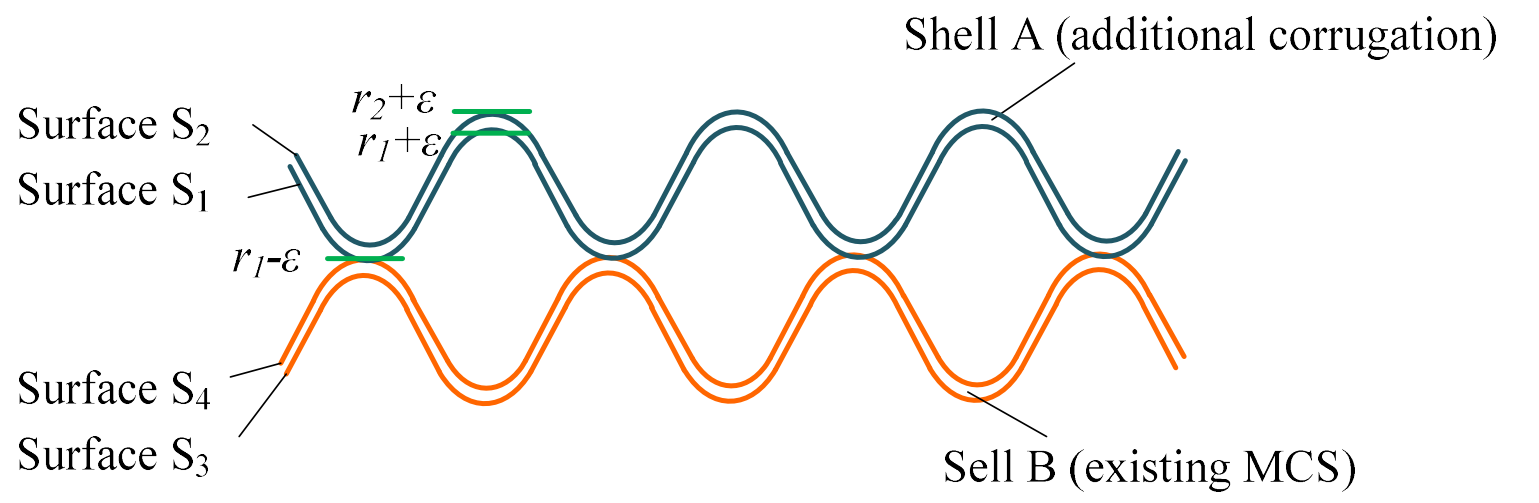

Figure 7. Scheme for the description of the mathematical model of double corrugation

Shell A is under the action of forces $P_{r}(\varphi, z), P_{z}(\varphi, z)$ applied to the outer surface $S_{2}$. The inner surface $S_{1}$ of shell A and the outer surface $S_{4}$ of shell B, except for lines (33), are free from loads. The inner surface $S_{3}$ of shell B and side surfaces $z= \pm l$ of both shells are also free from loads.

To determine the stress state of the shells under consideration, we use the above equations and relations of the theory of elasticity (12)-(14). In this case, the set $S$ is the union of surfaces $S_{1}, S_{3}$ and $S_{4}$, from which the lines (33) are subtracted.

According to the conditions of the ideal mechanical contact of shells A and B, the values $\sigma_{r r}, \sigma_{r \varphi}, \sigma_{r z}, u_{r}, u_{\varphi}, u_{z}$ at $r=r_{1}-\varepsilon-0, z=\frac{\pi}{a}\left(-\frac{1}{2}+2 n\right)$ are equal to the values at $r=r_{1}-\varepsilon+0, z=\frac{\pi}{a}\left(-\frac{1}{2}+2 n\right)$. The guide cosines $n_{r}, n_{z}$, which correspond to surfaces $S_{1}, S_{2}$, are determined according to formulas (23)-(24). The following guide cosines correspond to $S_{3}$ surface:

$$
n_{r}=-\frac{1}{\Delta}, n_{z}=-\frac{\varepsilon a \cos a z}{\Delta},
$$

and the following values correspond to $S_{4}$ surface: 


$$
n_{r}=\frac{1}{\Delta}, n_{z}=\frac{\varepsilon a \cos a z}{\Delta}
$$

where

$$
\Delta=\sqrt{1+(\varepsilon a \cos a z)^{2}} .
$$

According to the above method of assessing the stress-strain state of transport constructions made of corrugated metal structures reinforced with the proposed stiffeners, we will assess the stress-strain state in the following sections of the work.

\subsubsection{Comparative assessment of the stress-strain state of constructions with MCSs reinforced with the help of proposed design options}

To study the effectiveness of the proposed design solutions with the aim to increase the load-bearing capacity of transport constructions made of corrugated metal structures, we will take, as a basis, the design of the construction for the railway and highway of the first category in Kropyvnytskyi city. According to electronic source [15], the horizontal diameter of the construction is $9,23 \mathrm{~m}$, and the vertical one is 8,12 m (Fig. 8).

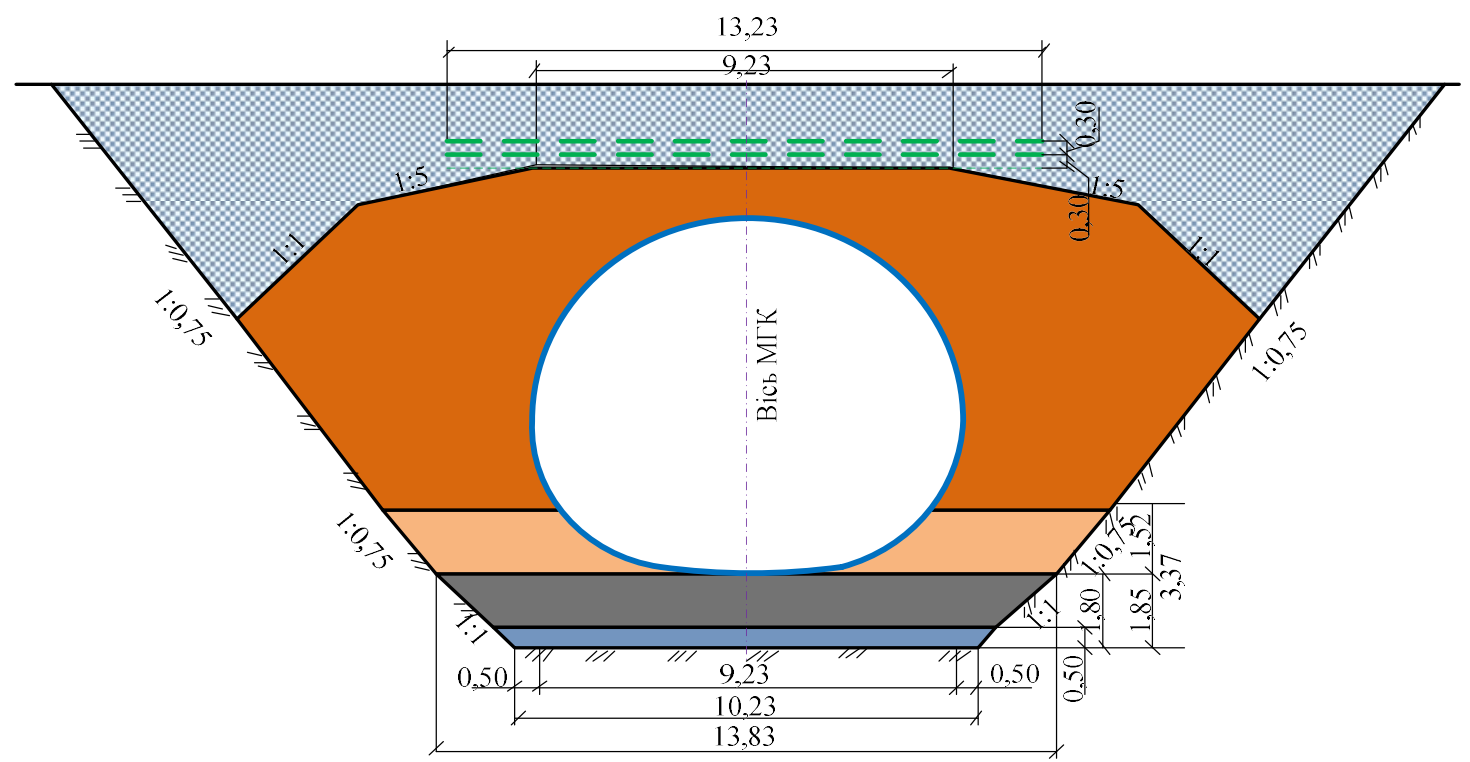

Figure 8. Cross section of the investigated construction with MCSs in Kropyvnytskyi city [15]

Metal sheets of the underpass are made of corrugated structures with wavelength and height of corrugation $380 \times 140 \mathrm{~mm}$ and thickness of $6 \mathrm{~mm}$ (Fig. 9). The modulus of elasticity of the metal pipe is $E=2,1 \cdot 10^{5} \mathrm{MPa}$, and Poisson's ratio is $v=0,25$. 


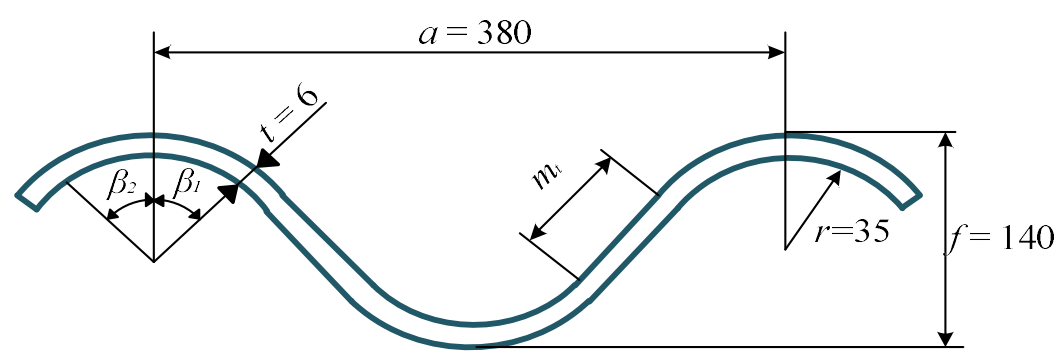

Figure 9. Geometrical parameters of the corrugated metal sheet

Physical and mechanical characteristics of the round stiffening rib and the reinforcing plate were taken as follows: modulus of elasticity $-E=2,1 \cdot 10^{5} \mathrm{MPa}$ and Poisson's ratio of the construction material $-v=0,25$.

Soil with the following physical and mechanical parameters was used as a backfill: bulk density $-\rho=1,9 \mathrm{t} / \mathrm{m}^{3}$, specific gravity $-\rho_{s}=2,65 \mathrm{t} / \mathrm{m}^{3}$, bulk density of soil $-\gamma_{c v}=19,0 \mathrm{kN} / \mathrm{m}^{3}$, soil moisture $-W_{n}=3 \%$ and the angle of internal friction $-\varphi_{c v, k}$ $=41,9$ degrees. The degree of soil compaction is 0,98 , and the modulus of elasticity is $E_{z p .}=24,0 \mathrm{MPa}$. The height of the backfill above the vault of the corrugated metal structure of the underpass is $2,57 \mathrm{~m}$.

The load from the own weight of the backfill soil, as well as the load from locomotive 2M62 are taken as the load. The results of equivalent forces arising from locomotive 2M62 are shown in Fig. 10.

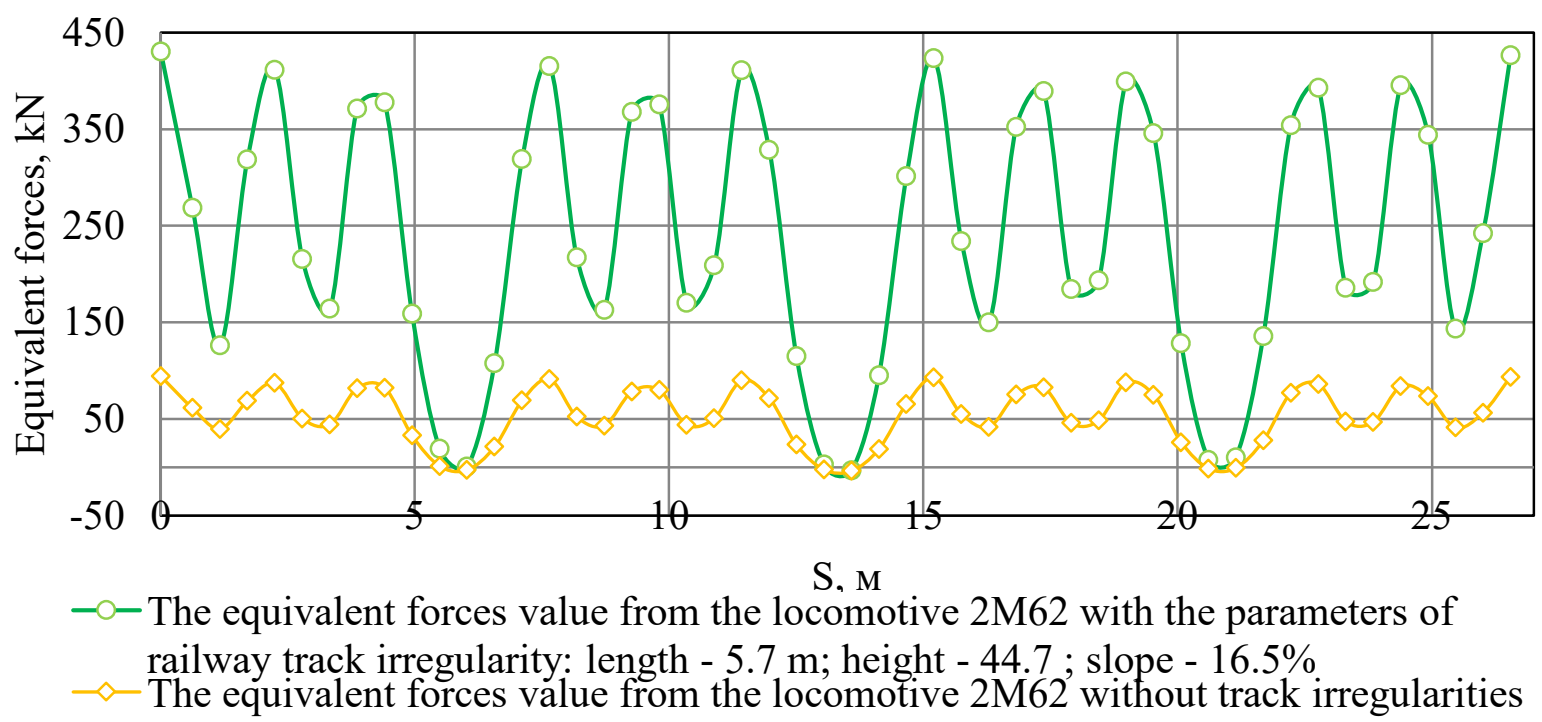

Figure 10. Distribution of equivalent forces from locomotive 2M62 at the value of the modulus of elasticity of an underrail base $92,1 \mathrm{MPa}$ 
The distribution of loads on sleepers along the track was obtained from the track calculations for strength [26] at the value of the modulus of elasticity of the underrail base equal to $92,1 \mathrm{MPa}$, and the geometrical unevenness formed during operation of the corrugated metal structure. The maximum value of the equivalent force is 423,6 $\mathrm{kN}$.

According to the obtained values of equivalent forces, we will evaluate the operational reliability of the design solutions made to increase the bearing capacity of a transport construction made of corrugated metal structures.

The calculation of the stress-strain state of the proposed design solutions to increase the load-bearing capacity of constructions with MCSs was performed by finite element modelling in licensed software Femap NX Nastran, in accordance with the recommendations given in [27-39].

\subsubsection{Investigation of the influence of corrugated sheet thickness on}

\section{increasing the bearing capacity of a transport construction made of corrugated metal structures}

Evaluation of the bearing capacity of a construction is carried out at metal corrugated sheet thickness 2 to $10 \mathrm{~mm}$ with a step of $2 \mathrm{~mm}$.

The developed finite element model consists of 39,504 elements, which are connected by 40,294 nodes. The simulation results in the form of stress and strain distribution that occur in the metal sheets of the construction are given in table 1.

As can be seen from table 1, the maximum stresses and strains occur in the vault of the structure. Thus, when the thickness of a metal corrugated sheet is $2 \mathrm{~mm}$, the strain is $110,4 \mathrm{~mm}$; at sheet thickness of $4 \mathrm{~mm}-26,09 \mathrm{~mm}$; at sheet thickness of $6 \mathrm{~mm}$ $-10,14 \mathrm{~mm}$; at sheet thickness of $8 \mathrm{~mm}-5,14 \mathrm{~mm}$ and at sheet thickness of $10 \mathrm{~mm}-$ $3,06 \mathrm{~mm}$.

The stresses that occur when the thickness of a metal corrugated sheet is $2 \mathrm{~mm}$ are $361.6 \mathrm{MPa}$; at sheet thickness of $4 \mathrm{~mm}-126,1 \mathrm{MPa}$; at sheet thickness of $6 \mathrm{~mm}-$ $64,15 \mathrm{MPa}$; at sheet thickness of $8 \mathrm{~mm}-39,99 \mathrm{MPa}$ and at sheet thickness of $10 \mathrm{~mm}$ $-28,5 \mathrm{MPa}$. 
Table 1

Stress-Strain State of MCS Depending on the Thickness of a Metal Corrugated Sheet of the Construction

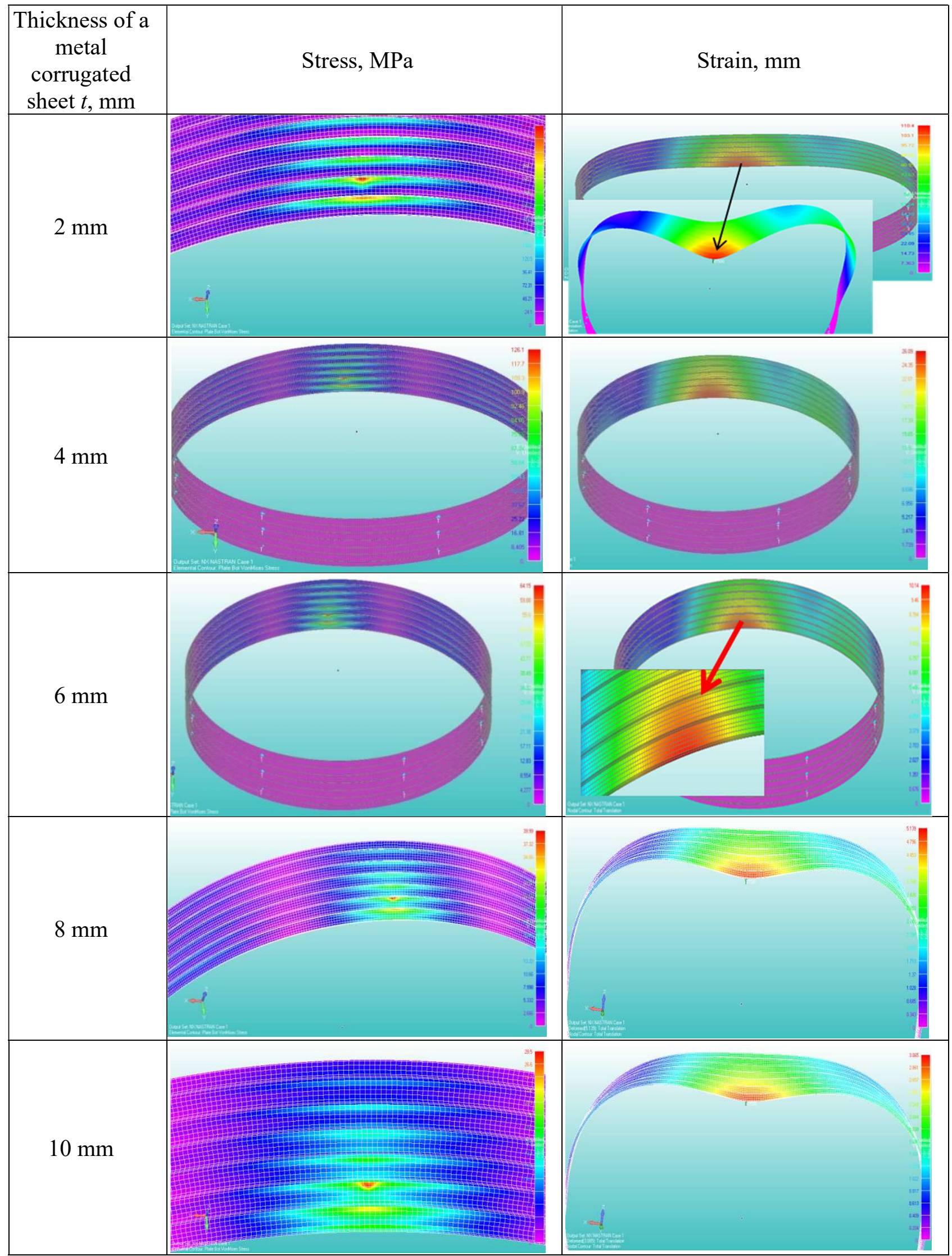


Thus, the thickness of a corrugated sheet has a significant effect on the stress distribution in the construction. With increasing sheet thickness, the stresses decrease, the dependence of stresses on the thickness of a corrugated sheet is nonlinear (Fig. 11).

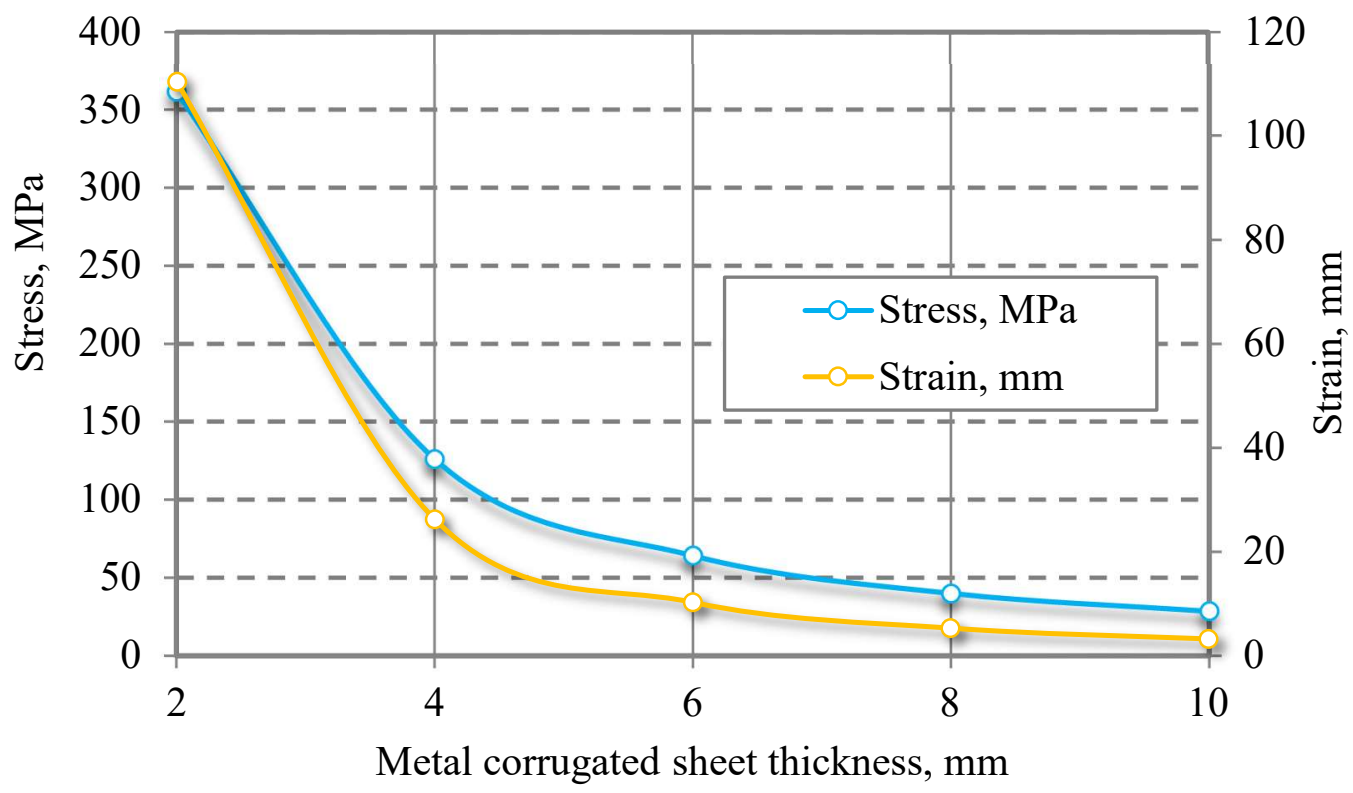

Figure 11. Dependence of stresses and strains on the thickness of a metal sheet

It is established that when the thickness of a corrugated sheet increases 5 times, the stresses decrease 13 times.

\subsubsection{Stress-strain state of a corrugated metal structure reinforced with stiffeners}

Let's consider the option of a metal corrugated pipe reinforcing at wall thickness of $6 \mathrm{~mm}$ with a round rod of $16 \mathrm{~mm}$ diameter, which is installed in the sinuses of the corrugated structure. The finite element model consists of 453,917 nodes and 146,586 finite elements (Fig. 12).

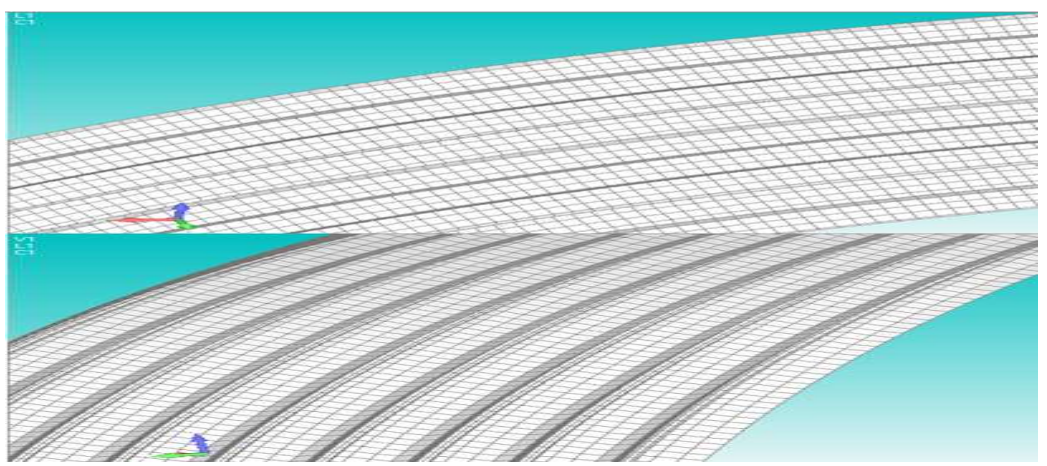

Figure 12. Finite element model 
The results of stress and strain distribution in the underpass construction are shown in Fig. 13.

When reinforcing a metal corrugated structure with installing additional stiffeners at corrugated sheet thickness of $6 \mathrm{~mm}$, there is a decrease in stresses in the structure compared to the structure without stiffeners. The maximum stresses that occur in the wall of a MCS, when using round stiffeners are 23,55 MPa, and the maximum strain $-2,92 \mathrm{~mm}$. In the case of strengthening the structure with stiffening plates, the maximum stress is $23,21 \mathrm{MPa}$, and the maximum strain is $2,07 \mathrm{~mm}$.

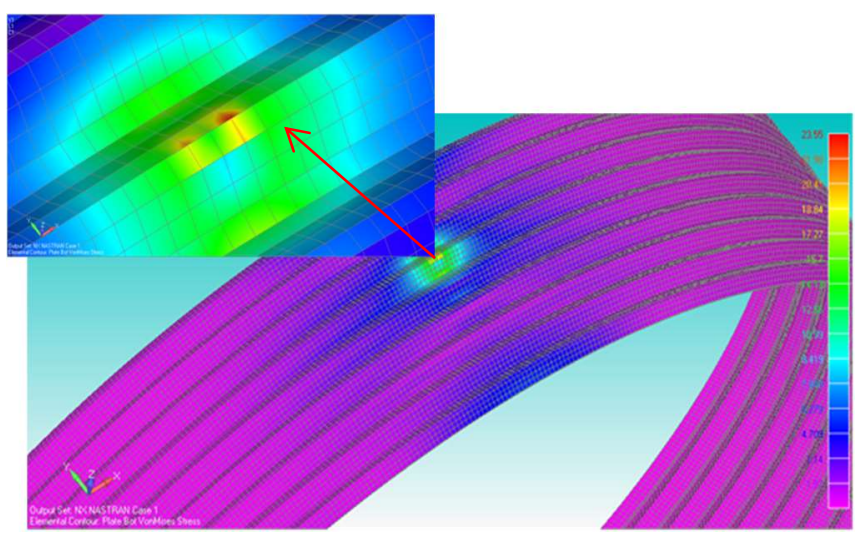

$a$

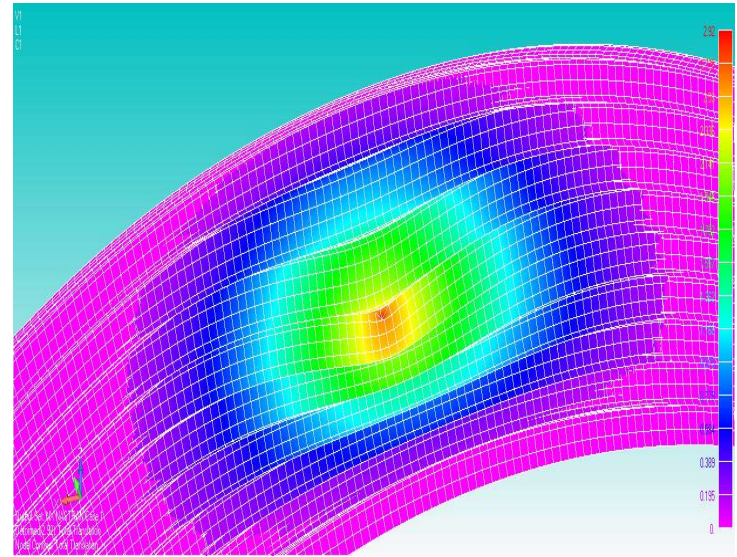

$b$

Figure 13. Stress-strain state of a transport construction with MCS when installing additional stiffeners: $\mathrm{a}$ - stress distribution; $\mathrm{b}$ - strain distribution

\subsubsection{Estimation of the bearing capacity of a metal corrugated structure at double corrugation}

The finite element model of a metal corrugated structure reinforced with double corrugation is shown in Fig. 14. The model consists of 413,946 nodes and 410,800 finite elements.

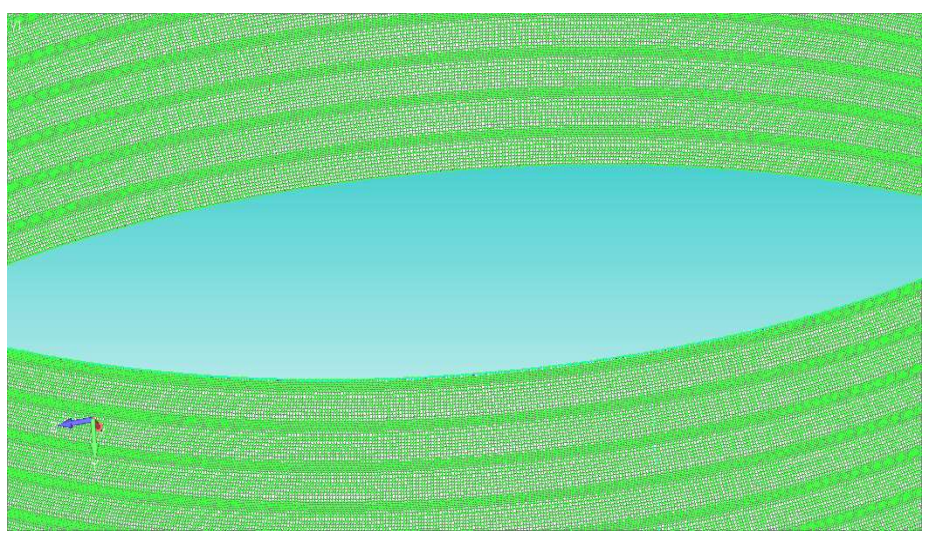

Figure 14. Finite element model of MCS with double corrugation 
The results of Mises stresses distribution in a metal corrugated construction reinforced with double corrugation are shown in Fig. 15.

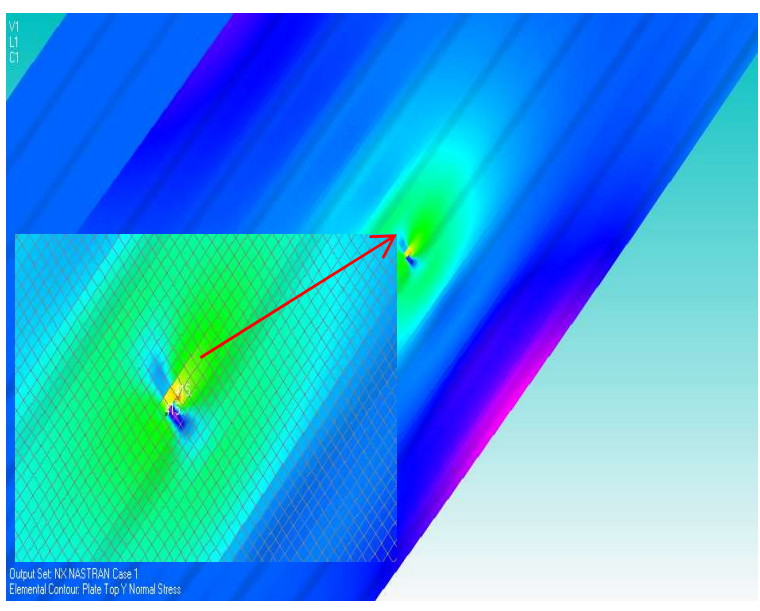

$a$

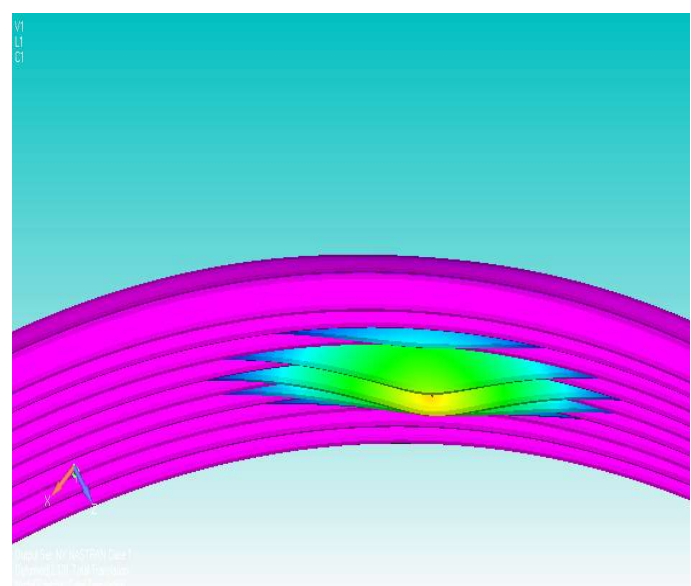

$b$

Figure 15. Stress-strain state of the transport construction made of MCSs with double corrugation: $\mathrm{a}$ - stresses distribution; $\mathrm{b}$ - strains distribution

As can be seen from Fig. 15, the maximum stresses that occur in metal sheets of the construction with double corrugation are $23,44 \mathrm{MPa}$, and the maximum strain in the vault of the construction $-2,13 \mathrm{~mm}$.

The results of the calculation of stresses in the structure with double corrugation at additional stiffeners use, which occur in the sheets of the construction, showed that the stresses are less than the allowable value of $235 \mathrm{MPa}$.

\subsubsection{Calculation results comparison of the stress-strain state of} transport constructions reinforced with additional elements

The results of the calculation of the stress-strain state of the MCSs depending on the design option of structural reinforcement are given in table 2.

The calculation of the bearing capacity of the construction in the case of pipe corrugation with its thickness of $6 \mathrm{~mm}$ showed that the stress is $64,15 \mathrm{MPa}$, and in the absence of corrugation at the same thickness of the structure - $286 \mathrm{MPa}$. Thus, corrugation increases the bearing capacity of the metal structure by 4,5 times.

The calculations show that the lowest stresses and strains occur in the design options where MCS bearing capacity is increased by double corrugation and additional stiffeners installation. The stresses that occur in the sheets during double corrugation 
are $23,44 \mathrm{MPa}$, and strains $-2,13 \mathrm{~mm}$. In the case of stiffeners installation in the form of round rod with diameter of $16 \mathrm{~mm}$, the stress is $23,55 \mathrm{MPa}$, and the strain is 2,92 $\mathrm{mm}$. When using stiffeners in the form of plates, the stress is $23,21 \mathrm{MPa}$, and the strain is $2,07 \mathrm{~mm}$.

Table 2

Comparison of Stress-strain States of Proposed Methods of MCS Bearing Capacity Increase

\begin{tabular}{|c|c|c|c|}
\hline № & $\begin{array}{c}\text { Option of MCS bearing } \\
\text { capacity increase }\end{array}$ & Stress $\sigma, \mathrm{MPa}$ & Strain $\varepsilon, \mathrm{mm}$ \\
\hline 1 & $\begin{array}{c}\text { Without sheet } \\
\text { corrugation } \\
\text { at thickness 6 mm }\end{array}$ & 286 & - \\
\hline 2 & Sheet thickness 2 mm & 361,6 & 110,4 \\
\hline 3 & Sheet thickness 4 mm & 126,1 & 26,09 \\
\hline 4 & Sheet thickness 6 mm & 64,15 & 10,14 \\
\hline 5 & Sheet thickness 8 mm & 39,99 & 5,14 \\
\hline 6 & Sheet thickness 10 mm & 28,5 & 3,06 \\
\hline 7 & Round rod stiffening & 23,55 & 2,92 \\
\hline 8 & Plate stiffening & 23,21 & 2,07 \\
\hline 9 & Double corrugation & 23,44 & 2,13 \\
\hline
\end{tabular}

At this level of loads on the transport construction with metal structure of $6 \mathrm{~mm}$ thickness and absence of corrugation as well as in the metal corrugated structure of 2 $\mathrm{mm}$ thickness, stresses exceed the allowable limit of metal elasticity $235 \mathrm{MPa}$.

In other design options, the stresses in the cross sections of MCSs in relation to the basic technical solution are reduced and do not exceed the yield strength of steel, thus, each of the proposed solutions is efficient in terms of preventing the transition of metal to plastic state.

However, the proposed design options require increased economic costs and complexity of work. To establish the most profitable and optimal design solution, it is necessary to conduct a feasibility study.

\subsubsection{Technical and economical evaluation of design solutions efficiency to increase the bearing capacity of metal corrugated structures}

To assess the effectiveness of the proposed design solutions, it is proposed to use a comprehensive efficiency index, which takes into account the technical characteristics of the proposed options for strengthening the construction with MCSs and economic components determined by the formula: 


$$
e=\sigma_{e}+E_{e},
$$

where: $\sigma_{e}$ - technical index of design solution efficiency which considers maximum stresses; $E_{e}$ - factor that takes into account the cost of the basic technical solution, the increase of the volume of construction and installation work and their cost.

The integrated approach provides the ability to take into account a number of factors that influence the effectiveness of the proposed technical solution. The following factors can be distinguished: change in the cost of building materials; increase in the cost of construction due to the use of additional materials; increase in labour intensity caused by additional volumes of construction and installation work during reinforcement, etc.

The technical index according to maximum stresses is calculated by the ratio of allowable stresses that do not exceed the elastic limit of the metal structure to the maximum stresses obtained in the calculation of the proposed design solution:

$$
\sigma_{e}=\frac{[\sigma]}{\sigma_{\max }}
$$

where: $\sigma_{\max }-$ maximum stress obtained at a specific design solution to increase the bearing capacity of the MCS; $[\sigma]$ - the allowable value of metal elasticity of the MCS; it is $235 \mathrm{MPa}$ for steel St3ps.

The coefficient, which takes into account the cost of the basic technical solution, the increase in the volume of construction and installation work and their cost, is determined by the formula:

$$
E_{e}=\frac{K}{K+k},
$$

where: $K$ - cost of metal structures of the basic technical solution, thousand UAH; $k-$ cost of additional materials for reinforcement of constructions with MCSы, thousand $\mathrm{UAH}$.

Determination of the coefficient increase in the cost of the construction $E_{e}$ is carried out in accordance with the average cost of structures and materials of domestic MCSs manufacturers. The cost of materials is given in table 3. The increase in costs due to additional reinforcement of the construction with MCSs, is taken equal to 7050 
$\mathrm{UAH} / \mathrm{t}$ at sheet thickness of $1 \mathrm{~mm}$.

Table 3

Technical and Economical Indices of Proposed Design Solutions

\begin{tabular}{|c|c|c|c|c|c|c|c|}
\hline Technical solution & $\begin{array}{c}\text { Metal } \\
\text { thickness, mm }\end{array}$ & $\begin{array}{c}\sigma_{\max }, \\
\mathrm{MPa}\end{array}$ & $\sigma_{e}$ & $K, \mathrm{UAH}$ & $k, \mathrm{UAH}$ & $E_{e}$ & $e$ \\
\hline $\begin{array}{c}\text { Without sheet corrugation } \\
\text { at thickness 6 mm }\end{array}$ & 6 & 286 & 0,82 & 6700 & - & 1,0 & 1,82 \\
\hline Sheet thickness 2 mm & 2 & 361,6 & 0,64 & 14150 & - & 1,0 & 1,64 \\
\hline Sheet thickness 4 mm & 4 & 126,1 & 1,86 & 28300 & - & 1,0 & 2,86 \\
\hline Sheet thickness 6 mm & 6 & 64,15 & 3,66 & 42450 & - & 1,0 & 4,66 \\
\hline Sheet thickness 8 mm & 8 & 39,99 & 5,88 & 56600 & - & 1,0 & 6,88 \\
\hline Sheet thickness 10 mm & 10 & 28,5 & 8,25 & 70750 & - & 1,0 & 9,25 \\
\hline Reinforced with round rod & 6 & 23,55 & 9,98 & 42450 & 847,27 & 0,98 & 10,96 \\
\hline $\begin{array}{c}\text { Reinforced with additional } \\
\text { plate }\end{array}$ & - & 23,21 & 10,12 & 42450 & 3500 & 0,92 & 11,04 \\
\hline Double corrugation & 6 & 23,44 & 10,03 & 42450 & 4245 & 0,91 & 10,94 \\
\hline
\end{tabular}

When reinforcing structures with additional stiffeners in the form of round rods are used, the efficiency is 10,96, when using stiffeners in the form of a reinforcing plate, the efficiency is 11,04 and with double corrugation $-10,94$.

The most effective technical and economic options for increasing the bearing capacity of MCSs are structural options for strengthening the metal corrugated structure by installing stiffeners and double corrugation. These design options provide high load-bearing capacity of MCSs and are, at the same time, the best options in terms of price/quality. 